\title{
SUFIJOS NOMINALES Y ADJETIVALES EN IBICENCO ${ }^{1}$
}

El primer problema que se nos plantea al emprender el estudio de los sufijos de una lengua viva, es el de su clasificación. Como observa el profesor Alvar, en El dialecto aragonés ${ }^{2}$, tanto la clasificación alfabética (que él adopta en ese libro), como la ideológica (que empleó en El habla del Campo de Jaca), tienen sus inconvenientes. La primera, aceptable en una gramática histórica en que se hace un estudio diacrónico partiendo de las formas originarias hasta llegar a los resultados actuales, tiene para nuestro caso el grave inconveniente de presentar uno tras otro, sin orden alguno, sufijos de valores miny distintos y que han sufrido suertes muy diversas. La agrupación por significados es mucho más lógica, pero presenta también dificultades considerables: por un lado, hay sufijo:; que tienen actualmente un valor muy distinto del que tuvicron en otras épocas, y, por otra parte, un solo sufijo asume con frecuencia funciones diversas.

1 Después de haberme propuesto tomar como tema de mi tesis. doctoral el dialecto catalán hablado en la isla de Ibiza, me encontré con que la abundancia de materiales y la cantidad de problemas planteados exigian una gran cantidad de tiempo y espacio, $y$, de acuerdo con el profesor Badía, decill consagrar la tesis al "léxico ibiccnco", sin renunciar, sin embargo, al propósito de hacer un estudio completo del dialecto, del que la tesis constituirá una parte. İl presente trabajo es parte de otro capitulo (el de formacioun de palabras). Por ello me ocupo raramente de la etimología de los radicales, que lie de tratar en la tesis.

2 Mr. Alvar, El dialecto aragonés, Gredos, I953. Madricl, p. 25,4, nota 5 . 
En el presente trabajo se hace el estudio de los sufijos del ibicenco partiendo de otra base de clasificación: la de la vitalidad de los sufijos, a la que Meyer-Lübke, en su gramática de las lenguas románicas, dedica considerable atención, y cuya importancia, puesta de relieve por $\mathrm{Nyrop}^{1}$ y por todos los trabajos posteriores, me ha sido presentada con nuevas luces en el curso Ausgevählte Kapitel aus der Wortbildung und Bedentungslehre des Französischen, dado por el Prof. Steiger en $Z$ ürich, durante el semestre de verano de 1956 .

Tratándose de un estudio dialectal, esencialmente sincrónico, nos interesará primordialmente saber cuáles son los sufijos vivos hoy, de los que el hablante pueda echar mano en todo momento para cualquier formación. En segundo lugar, importa estudiar los restos de sufijos actualmente improductivos que se encuentran en la lengua.

De acuerdo con ese criterio, y siguiendo al Prof. Steiger, he clasificado los sufijos en tres grupos, de menor a mayor inportancia:

a) Sufijos muertos: los que han perdido su valor como tales y son hoy completamente improductivos; en algunos casos resulta incluso difícil determinar el valor originario del sufijo.

b) Sufijos paralizados: los que, habiendo gozado de fuerte vitalidad hasta épocas tardias, son percibidos por el hablante como sufijos de un valor determinado, pero no tienen fuerza ya para nuevas formaciones, o la tienen muy escasa.

c) Sufijos vivos, que se encuentran en plena fuerza creadora y pueden formar derivados de cualquier raíz ${ }^{2}$.

A lo largo de la vida de un sufijo, ciertas formaciones que

1 Grammaire historique de la lang!e française, t. III, Copanhagus 1908 .

2 En realiclad, esta división se encuentra ya insinuada por Nyror, Gram., III, párrafos 37-39, que los distribuye en vivos y muertos, pero advierte que un sufijo puede estar vivo en una palabra sin ser productivo (párr. $3^{8}$ ), o ser perfectamente reconocible y presentar una idea neta al espiritu sin ser productivo (párr. 39), lo cual viene a corresponder a la idea de paralización. 
empezaron siendo derivadas con el valor general del sufijo, se concretan en un sentido especial; esta concreción puede darse en cualquier momento de la vida de la lengua, y así hay especializaciones que se dieron ya en latín vulgar, y pasaron de este modo a las lenguas románicas, y otras que son recicntes. Por lo tanto, al estudiar un sufijo paralizado, hay que considerar también los fósiles de un valor primitivo del sufijo, luego perdido, y al estudiar los vivos se deben tener en cuenta las especializaciones de sentido del valor actual, y los fósiles de otros valores. Así, el sufijo aumentativo -às, -assa, en plena vitalidad (donassa, 'mujer grande'), presenta especializaciones como la de vidassa (ell principio 'gran vida,'), que se ha concretado en ibicenco en el sentido de 'fiesta ruidosa, juerga', y se encuentra fosilizado en voces como fogassa < foc a cea.

Con frecuencia es difícil determinar si un sufijo está completamente muerto o sólo paralizado, o si otro que parece paralizado no conserva una cierta capacidad creadora. En algunos casos sería preciso hacer un estudio especial para determinar a qué categoría pertenece el sufijo.

Tratándose de un estudio dialectal, aparecen como paralizados, o no se mencionan, sufijos como -able, -ible, -ista, -isme, etcétera, que en la lengua literaria o en sectores cultos de la sociedad gozan de plena vitalidad.

Los restos de sufijos totalmente muertos son muy antiguos en la lengua, y por lo general comunes a todo o a una bucna parte del dominio idiomático. Por cllo se consignan aquí, acompañados de ejemplos, pero sin entrar en detalles. Al tratar de sufijos vivos o paralizados, se reproducen únicamente los fósiles y especializaciones interesantes para el ibicenco, prescindiendo de los que son generales o aludiendo a ellos muy de pasada. (Para completar la información para cl catalán en general, consúltese F. de B. Moll. Gramática histórica catalana Edit. Gredos, págs. 265-299.)

I os sufijos muertos y paralizados se presentan por simple orden alfabético de resultados. En el capítulo de los vivos se ha procurado hacer una subagrupación por significados. 
A) Sufijos muertos.

I. $-A C<-a c c u$, sufijo de origen oscuro que aparece, siempre como mucrto, en español (verraco, sobaco), gascón (flaunak, poudac), aragonés (bušaco, alberitaco $)^{1}$ y catalán: pinyac, xerrac, fullaca, etc. Citemos además para el ibicenco ronsac 'cereal parecido a la cizaña'; llimac 'limo verde que se forma en los estanques'; xodrac (catalán general sotrac) 'sacudida dada por un carro'. Resulta difícil determinar el valor originario de este sufijo, y a veces hasta su misma existencia; así, llimac puede proceder de una forma latina $* 1$ i m a$\mathrm{cu}, \mathrm{y}$ xodrac y xerrac pudicran muy bien ser formaciones onomatopéyicas. En fullaca 'hojarasca', sin embargo, parece evidente que nos hallamos ante un sufijo -aca con claro valor colectivo-despectivo.

2. - AINA (ájné), que se considera el resultado mozárabe del sufijo -a $g$ in e (cfr. en algunas regiones del catalán y en aragonés plantaina, farraina), dió en catalán continental cierto número de derivados, todos ellos con matiz de frivolidad o insustancialidad (V. Moll, Gr., § 364). Muy escasos restos en ibicenco: xerraina 'quien todo lo cuenta, que no sabe guardar un secreto' (seguramente deformación de $x e$ rraire; una deformación paralela ha dado en Nallorca guixaina, de guixaire 'yesero'); llepugaina (de llepar 'lamer' < 1 a pp a re , REW, 4905); llemiscaina (de llenisc, 'limo, pringue'); llagastaina, los tres con significado de 'adulación, alabanza exagerada e hipócrita'; golaina o golosaina 'golosina'.

3. -AIRE (áiré) < -a to r en su forma de nominativo, de influencia provenzal, se mantiene vivo en catalán central y occidental, pero está muerto por completo en valenciano y balear. Documentado en ibicenco: xerraire 'hablador' y tonaire 'aparejo para pescar atún'.

4. ${ }^{\circ}$-ANY, -ANYA (-ån) < -a ne u , muerto hoy en catalán lo mismo que en español y francés. Aparte fósiles comu-

1 Cfr. Alvar, Dial. Ar., párr. I 38. 
nes a diversas lenguas, como muntanya, fogany'a, etc., se encuentra en algunos derivados conservando su valor de aproximación o peyorativo: durany 'callosidad', carrerany 'sendeIo', tirany 'camino de cabras'.

5..$^{\circ}$-ARD $<$ germ. -ha $\mathrm{t} t$, generalizado como sufijo en italiano y francés (en que tiene aún hoy plena vitaliclad), de donde pasó a la Península Ibérica, nunca tuvo en catalán gran vitalidad. Aparece en los galicismos covard 'cobarde', y bastard (que además de la significación normal de 'bastardo' tiene en ibicenco la de 'ordinario, de escaso valor': p. ej., "una roba bastarda川) y en moscard 'mosquito', usado sólo en las Baleares.

6. - ASÓ ( $z z o ́ o)<-a$ t i o n e, que dió una larga serie de derivados en catalán antiguo (V. Moll, Gr., §378), se conserva en el ibicenco clavasó "clavos de cabeza ancha para reforzar la suela de las alpargatas'.

$7 .^{\circ}$-ASTRE, -ASTRA (-ástre) <-a s te r produjo cn catalán, como en las otras lenguas tománicas, una serie des formaciones: madrastra, germanastre, pollastre, etc. (pero tuvo menos vitalidad que en francés). En Ihiza figura además en la voz embenastra (émbénástré) 'vendaje mal hecho', en que no parece tratarse propiamente del sufijo, sino probablementc de una etimología popular, por influencia de banastra 'canasta' (del latín ca nistrum, con cruzamiento del gálico be n n a ) $)^{2}$.

8. - ATGE (ázé) <-a g in e se encuentra sólo en fóciles del latín: plantatge, farratge.

9. ${ }^{\circ}-\mathrm{EC},-\mathrm{EGA}\left(\dot{\varepsilon}^{k},-\dot{\varepsilon} g \dot{b}\right)<-\mathrm{e} \mathrm{c}$ u, sufijo dudoso ${ }^{2} \mathrm{mu}-$ cho más abundante en español (-iego) que en catalán. Aparcee en fredolec 'friolero' (usado en las Baleares y en parte del catalán occidental); marrec 'cordero enfermo', y como adjetivo 'malo, de escaso valor' (en catalán continental se usa como

1 Cfr. J. Coronunas, Diccionario critico etimológico de la lengua castellana, Madrid. Edit. Gredos, t. I, P. 384.

2 Cfr. RomLF, Beiträge zur Kenntnis der Pyrenäenmundarton, RLi.R, VII, p. I43. 
sustantivo, con significado de 'chico'); xubec (cat. occid. sobec) 'sueño corto y poco profundo'.

Io. -ELL, -ELLA $(-\hat{e} \underline{i},-\tilde{e} i \hat{i} \dot{e})^{1}<-\check{i} \mathrm{c} \mathrm{u} 1 \mathrm{u}$. Aparte fósiles comunes a todo el catalán, (orella, abella, etc.), hay que citar en ibicenco solell (sulëi) 'sol' (es decir, 'rayos solares'; el astro solar se llama sol), conocido también en Mallorca y Menorca - sobre todo en su forma diminutiva solellet-, pero usado sólo esporádicamente, mientras en Ibiza es la única forma usada para 'rayos solares'. Devessell (dévésêi ), catalán general devessall, 'avalancha, chorro', presenta adaptación del mismo sufijo.

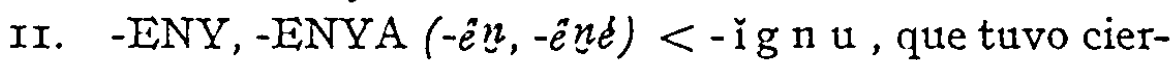
ta vitalidad en catalán para formar adjetivos de materia a menudo sustantivados, es muy escaso en Ibiza. Aparte fósiles sustantivados (como pedreny, espardenya, de uso general), se encuentra en algunos adjetivos como bultereny 'mal vestido, descuidado'; cagueny 'avaro'.

I2. $-\mathrm{IA}<-\mathrm{ia}+\mathrm{gr}$. $\{\alpha$, fosilizado en malaltia 'enfermedad' $y$ que ha dado numerosos derivados en otras regiones catalanas (Menorca, por ejemplo), como mestria, etc., ha sido prácticamente improductivo en Ibiza como sufijo independiente, pero se conserva en la forma compuesta -eria, viva actualmente en catalán, como en las otras lenguas románicas.

I3. -ILL, -ILLA ( $i, i \delta)<-i c u l u$ se conserva sólo en fósiles comunes a todo el dominio catalán: conill (kuni); llentilla (léntíe); clavilla (klèvíé), pero no se encuentran en ibicenco aplicaciones románicas del sufijo (del tipo del catalán continental cordill, etc.).

I4. - IT $<$-ittu ( $>$ esp. -ito) se encuentra en Ibiza, como en el resto del catalán, únicamente en los fósiles cabrit, mosquit. En cambio, -ittu > -et, (francés -et, italiano -etto), vivísimo como sufijo diminutivo.

I5. -OC, -OCA (Q́k, $\left.Q^{\prime} k e^{3}\right)<-$ o c cu, sufijo probable-

1 Recuérdese que en el baleárico se pronuncia $y$ la $l l$ procedente de yod (y $l$ la de $l l$ latina), y que la $e$ procedente de $\bar{e}, \bar{l}$ latinas tónicas suena $\dot{e}$. 
mente ibérico que presenta las mismas dificultades que -a c c u y ha dejado en catalán, como en español, algunos fósiles. Usados en Ibiza: badoc 'mirón, papanatas' y bajoca 'vaina de legumbre'.

I6. -'OL, -'OLA ("ut, $\left.{ }^{*} u l^{3}\right)<-\breve{u} 1$ u aparece fosilizacio en còdol< cotulu, 'piedra'; pèsol < p is u 1 u, y se encuentra en una serie de voces de etimología oscura: mirgula. 'colmenilla (hongo)'; péntol 'pedazo; trozo de piedra'; rétol 'grieta de una pared' (¿deformación de crètua?); vérbol 'empeine (enfermedad de la piel)'. Además en gínjol < ziziphus (REW, 9627, Corominas, DCECast. s. v. azufaifa), con terminación inexplicada. Finalmente, por adaptación de una terminación conocida a palabras que resultan extrañas, se encuentra en bonitol 'bonito (pez)' y ninjol 'nicho' (acaso por influencia directa de ginjol).

I7. -UA, terminación de origen desconocido que aparece en el catalán general corrua y menjua (Moll, Gr., § 430), usado este último en Ibiza en sentido de 'piojos', y en el ibicenco gasua (gézíé) 'niña'.

I7. $-U C,-U G A<-u c u$, que ya en latín tuvo escasa vitalidad, se encuentra en unas pocas voces, todas ellas del catalán general o de área muy anuplia: poruc 'miedoso'; peiic 'calcetín'; ballaruga 'peonza pequeña'; badaluc 'mirón, papanatas'. Ira forma tallaruca (tééérîké) 'recorte, rebauadita' supone una forma $-4 \mathrm{c} \mathrm{cu}$, como el aragonés peduco, tallamuco (V. Alvar, Dial. Ar., § ISI).

\section{B) Sufijos paralizados.}

r. $^{\circ}-\bar{A},-$ ANA $<-a n u$. Aparte algunos fósiles de derivados adjetivales latinos, como solana 'pared hecha en la pendiente de un monte'; clariana 'pequeña abertura por la que pasa la luz'; corçna 'rama gruesa de la cepa', este sufijo ha servido en catalán, como en las demás lenguas románicas, para la formación de gentilicios (catalit, valencià, vigatì). Iin Ibiza se usa para gentilicios de fuera de la isla, pero no ha 
dado derivados autóctonos (los cuales se forman con -er, $-e n c,-i)$.

Ira forma ibicenca afartano (éfértaim) o afartan 'glotón' parece formada a initación de formas españolas, pues si fuera autóctona sería afartà.

2. $-\mathrm{AL}<-\mathrm{a} 1 \mathrm{e}$, paralizado hoy, ha formado algunos acljetivos de pertenencia, como los que formó en latín (garroics bovals "clase de algarrobas grandes, y revueltas como los cuernos de un buey'), y ha dejado una serie de fósiles del latín, como majoral (mizurát) 'aparcero'; embornal 'imbornal' $\left(<^{*} \text { ombrinale, según Joan Coromines }\right)^{1}$, y ha dado en catalán, como en las demás lenguas románicas, numerosos derivados, con diversos valores. Pueden citarse, como especialmente interesantes para el ibicenco: $a$ ) entre los de piezas de vestir: corporal 'corpiño', documentado en catalán antiguo; b) de instrumento: confessional 'confesonario'; c) indicador del lugar en que abunda una planta, tenemos sólo figucral, siendo en los demás casos stustituído por -ar; verinal 'lugar empantanado por exceso de lluvias, manaintial' tal vez sea derivado de vert 'veneno' con este sufijo locativo; $d$ ) aumentativo: ullal (uiát) 'agujero al fondo de un depósito de agua', toal (tuál, derivado de tou) 'masa de agua (de una cisterria, ctcétera.)'.

Brindals, 'obenques, cuerdas que van desde el palo de la barca a la amurada', parece una forma noderna e importada (¿tal vez barandales?); pero no he encontrado su equivalente en español ni italiano.

3. $.^{\circ}-\mathrm{AI}, \mathrm{L}\left(-a_{i} i\right)<-a \mathrm{c} \overline{\mathrm{u}} \mathrm{l} \mathrm{u}$ ha tenido en catalán mayor fucrza que en español (-ajo) y en francés (-ail), pero está actualmente paralizado. Aplicado a radicales verbales ha dado: a) Nombres de instrumento: ventall 'abanico', sonall 'badajo de la campana'; raspall 'escoba rústica hecha de ramas'; escombrall 'escoba para limpiar el horno'; entrepussall 'obstá-

1 En Homenatge a Antoni Rubió i Lluch, vol. III, Barcelona, I936, p. $29 \mathrm{x}$.

2 Cfr. Diccionari Catald-Valcncid-Balear, s. v. 
culo que hace tropezar'; tapall 'tapas de cama'. b) Nombres que indican la acción misma del verbo o su resultado: comenf̧all 'principio'; afegitall 'añadidura'.

Aplicado a radical nominal, con valor aproximativo, 's raro en ibicenco: forcall 'horca de aventar'.

No aparece claro el valor del sufijo en rogall y gargall (usados en todo el dominio catalán), ni en escarrotall 'mequetrefe'.

Forrall 'cerrojo' (al parecer usado sólo en Ibiza) no es un caso de sufijación, sino una regresión de forrellat ( $f u \bar{r}$ é $\mathfrak{i}^{a}$ át), que es la forma usual en todo el dominio catalán.

$4^{\circ}$-ANÇA (-insé) $<-$ a n t i a, improductivo cll catalán como en español en su forma popular, por haber sido desplazado por la forma culta -ància, se encucntra en alegrança 'alegría'; folgança 'alegría, diversión'; contança 'cuento, narración', y con forma plural en matances 'matanza del cerdo' y casances 'casamiento; boda'.

5. ${ }^{\circ}$-ATGE $\left(-a^{\prime} z\right)<-a$ t i c u presenta fósiles del primitivo valor latino (derivado del de adjetivo de pertenencia), como companatge 'lo que se come con el pan'; llengatge 'lenguaje, idioma'; missatge 'encargo'; oratge 'viento suave'; vilatge 'pueblo', y formaciones más modernas con valor colectivo: garbatge 'conjunto de gavillas'; relatge 'raíces de un árbol'; romiatge 'rumor ${ }^{1}$.

Aplicado a un radical verbal, to tenemos en armatge 'cuerda que forma el borde de la red'.

6. ${ }^{\circ}$-DAT (catalán general -tat) $<-t$ a t e, formador de sustantivos abstractos, vivo como cultismo pero paralizado en la lengua popular. Se encuentra en fósiles latinos como claredat 'luz'; autoridat (usado también en Ibiza con sentido de 'autorización'), y en algunas formaciones nuevas: fesedat 'debilidad, indisposición' (derivado de fes < fiss um, part. de $\mathrm{f}$ in dè r e ); estuguedat 'asco'.

Aplicado a nombres de persona (derivados de adjetivos

1 Ver más usos y ejemplos para el catalán general en MoLt. Gr., parr. 377 . 
latinos) tiene, adcmás del abstracto, valor colectivo: germandat 'conjunto de hermanos'; vesindat (vézindát) 'conjunto de vecinos'.

$7 .^{\circ}$-EA (ée), terminación de valor colectivo matizado con frecuencia de sentido peyorativo, que se encuentra en todas las Baleares (pero está actualmente paralizada): mallorquín y menorquín lulea, al lotea, caninea; ibicenco llurea, perxenea; brusinea, 'llovizna'; al lotea, boixerea 'multitud de niños' (boix $=$ 'niño' en ibicenco). Parece proceder del sufijo francés -ée $<-\mathrm{a} t \mathrm{a}$. Por lo menos, llurea 'casta, raza' procede del francés livrée. Perxenea (péršennéé) 'casta' parece deformación popular de progenie influída por llurea.

8. -ELL, -ELLA (él en que se ha perdido la noción del sufijo (cadella 'perra'; canella 'espinilla'; capell 'sombrero'; gonella 'vestido'; palmell 'palma de la mano'), y en numerosas voces - principalmente nombres de instrumento- en que conserva el carácter diminutivo: caveguell 'escardillo'; fornell 'fogón'; portell 'abertura hecha violentamente en un muro'; carbonell 'pedazo de carbón'; correntella 'carrera corta y animada'.

Aparece usado como simple sufijo diminutivo en tramuntanella; xeloquell; mitjornell 'viento suave (de1 N., del SE, del S.); cordella 'cuerda delgada'; escabotell 'rebaño pequeño'; pero en la actualidad está completamente paralizado, sustitufdo en Ibiza por la forma -illo, de introducción castellana.

Aplicado a radical verbal indicando el resultado de la acción se encuentra en escarxell (éskér rẹ́̂l) 'miga; salpicadura' (derivado de escarxar 'aplastar', de formación onomatopéyica).

Por cambio de sufijo lo tenemos en gratella 'escozor, ganas de rascarse' y en morenells 'aparejo para pescar morenas'.

$9^{\circ}$. -ENÇA (énsé) <-e $11 \mathrm{tia}$, que aparece en herença, aparença, está hoy completamente paralizado en su forma popular, suplantado por la culta -ència.

ro. -̇S, -ESA (ês, êzz) <-e n s e. Aparte algunos fósiles (pagès, etc.) y formaciones de época primitiva (marès 'arenisca'), ha servido en catalán como en las demás lenguas románicas para la formación de gentilicios. En Ibiza se usan 
los propios del catalán general (maonès, aragonès, etc.), pero no existen derivados locales con este sufijo.

II. -ESA $(-\hat{e} z \hat{e}<-$ it i a, ha tenido gran vitalidad para la formación de sustantivos abstractos usados en todo el dominio catalán (jovenesa, vellesa, etc.), pero hoy es improductivo. Son de notar, como propias del ibicenco, debilesa 'debilidad' y estuguesa 'asco'.

I2. -ESC, -ESCA (êsh, -ểske) <-ïscu ha dcjado en catalán, como en todas las lenguas románicas, numcrosos derivados (cavalleresc, moresc, etc.), pero está completamente paralizado. Es interesante la forma (garrova) panesca, con que se designa en Ibiza una clase especial de algarroba.

13. -EST (ést), -ESTA < -est is (en adjetivos latinos de pertenencia, como agrestis, coelestis) aparece conservado, al parecer sólo en Ibiza, en denominaciones de diferentes clases de tierra: arenest o terra arenesta 'arenisco, tierra arenosa'; argelest o $t$. argelesta 'tierra arcillosa'; blanquest o $t$. blanquesta 'tierra blanquecina, muy productiva'; vermellest 'tierra rojiza'; bardalest '(terreno) empinado'. Como fósil de este sufijo tenemos el catalín antiguo forest $<\mathrm{fo}$ restis ${ }^{1}$.

En Mallorca se conserva aún otro resto sustantivado, baixest 'declive, vertiente', derivado seguramente de baix 'bajo' con este sufijo, como ya propuso Spitzer ${ }^{2}$ (aunque no es necesario pensar en una influencia especial de forest) : la terminación -est debió hacerse productiva, ayudada indudablemente por las relativamente numerosas formas derivadas de adje-

1 Cfr. Wartburg, FEW, III, 709-Io, que lo presenta como abundantemente documentado a partir del año 648 . Wartburg consider- lorestis como derivado de foras o de for um. CoromNAS (DCE Cast., II, 543), siguiendo a GAMnLscheg (Romania Germanica, $I, 2$ I2), se inclina por un fráncico for his $t$, colectivo de for a h 'pino'. Sea cual fuere el origen de su raiz, es indudable que forest deriva directamente de este forest is 'bosque del rey'.

2 LEO SPITZER, Lexikalisches aus dem Katalanischen und don übrigen iberoromanischen Sprachen, Genève. Olscliki, I921. 
tivos que en latín terminaban en -e stus (honest, molest, modest, etc.), lo que permitió unas pocas formaciones adjetivales posteriores, como xalest 'alegre' (der. de xala 'diversión') y ferest 'temible, terrible' (derivado de fer 'fiero' < f è r u s), conocidos en todo el dominio catalán. Actualmente está por completo paralizado. Sólo el hecho de que el hablante tiene clara conciencia de que se trata de formas derivadas, ha determinado que se incluya -est en este grupo y no en el de los sufijos muertos.

I4. -IBLE $<-\mathrm{i}$ b il $1 \mathrm{e}$, más o menos vivo hoy para formaciones cultas, está totalmente paralizado en el habla popular. Se consigna aquí (en contra de lo que se hace con -able, -isme, etc.), porque al lado de voces propias del catalán general (possible, terrible, etc.) hay algunas típicas de Ibiza, o por lo menos de área muy restringida: passible 'bastante bueno, que puede pasar' (usado también en forma diminutiva: passiblet), ofendible 'ofensivo, molesto'. [La solución -ivol< $<-i$ b i $1 \mathrm{e}$ ha dejado sólo algún raro representante, como manegivol 'fácil de manejar'.]

I5. -IM $<-i \mathrm{~m}$ e $\mathrm{n}$, que ha tenido en catalán cierta vitalidad para formar derivados con valor colectivo o intensivo y la mantiene en mallorquín y menorquín, es escaso en ibicenco: ruixim 'llovizna'; renyolim 'regaño fuerte'; fotim (y sus derivados fotimet, fotinó) 'chico travieso' (der. de fotre).

I6. -INA $<-i n$ a . Aparte fósiles de formas latinas conservados en todo el dominio catalán (gallina, etc.), y de la voz ibicenca catralina 'especie de cardo' que tal vez proceda de *c a r d u e 1 i n a con deformación popular del radical, se ha usado en catalán para formar sustantivos que indican intensificación moderada de lo expresado por el radical: continuo, pero poco, o mucho pero lentamente. Se encuentra aplicado a radicales verbales de participio, o a radicales nominales (en este caso generalmente en combinación con otro sufijo); típicos del ibicenco son cremadina 'producto de la combustión de un hormiguero', fanguerina 'barro'; sanguerina 'sangre que brota débil pero continuamente'; ventolina 'viento suave'.

I7. -ÍS, -ISSA $<-i \mathrm{c}$ i u, -i i iu, aparte fósiles de uso 
general (como pants, canyis), ha dado derivaciones con valor diminutivo (carbonís, 'carbonilla'; narissos 'ventanas de la nariz') o despectivo (pellís 'harapo, guiñapo'). Aplicado a un radical de participio, formando adjetivos que indican facilidad o tendencia a la acción, se encuentra en solladís (suyédís) $\left(<{ }^{*} \mathrm{~s}\right.$ u $111 \mathrm{a}$ r e ) 'que se ensucia fácilmente'; (terra) conradissa 'cultivable'.

I8. -ISSA $<-i$ cia. Aplicado a radicales nominales, presenta formas usadas en todo el dominio catalán, como pallissa, 'pajar cubierto'; frontissa 'bisagra' (de *f r a c t it i a , con radical modificado por front). Aplicado a un radical de participio, formó derivados de acción intensa: llampadissa 'relampagueo'; nyeuladissa 'concierto de mat1lidos'; sacsadissa 'traqueteo'; posadissa 'acción de poner'; tremoladissa o tremolissa 'temblor intenso'. Hoy está paralizado, sustituído por -ada.

I9. -ISTA, como -isme, -able, etc., es un sufijo culto, carente de vitalidad en la lengua poptular. Es cle notar, sin embargo, como dato curioso, que los habitantes del pueblo ibicenco de San Carlos se llaman carlistes (además de carlins y carliners), seguramente denominación moderna formada a imitación de carlista 'partidario del carlismo'.

20. -OI, -OIA (Q́i, Q́yé). Aparece en ibicenco aplicado a unos pocos radicales nominales, con valor diminutivo-afectivo: caminoi 'caminito'; sangueroi o sangueroia 'sangre que brota en poca cantidad'. Aplicado a radicales verbales de infinitivo indica, también con matiz afectivo: a) el resultaclo de la acción: renyaroi o renyaroia 'regaño'; mesclaroi 'mezcla'; b) instrumento: lligaroi 'cordón'; jugaroi 'juguete'; c) agente: palparoi 'remolón' (der. de palpar). Las formas derivadas de verbos son rarísimas fuera de Ibiza; citemos, sin embargo, penjoll o penjaroll (en ibicenco pènžérói), corricnte en todo el catalán. En cambio, en el catalán general son numerosas las formas derivadas de nombres y también otras derivadas de adjetivos, como alegroi, frescoi, bonicoi, menudoi, etc. (con i final en todos los dialectos), que en ibicenco presentan siempre $o$ cerrada. También en derivados de nombres y adjetivos 
cita Rohlfs ${ }^{1}$ el mismo sufijo en el gascón pulòy, mistòy, chicoy, etcétera.

El origen de $-Q i$ no se ve claro. Rohlfs considera improbable el origen vasco propuesto por Meyer-Lübke en Das Katalanische e insinúa la posiblidad de una forma salida del lenguaje infantil. Por lo que hace al catalán, uno siente la tentación de relacionar $-Q \dot{i}$ con el sufijo latino $-\breve{u} \subset u \in 1 \mathfrak{u}$, a pesar de las dificultades que ello plantea. La relación parece apoyada por la distribución geográfica de $i$ y $l$ final en el caso de penjaroll; queda pendiente, sin embargo, el problema de la 0 , que debería ser cerrada ${ }^{2}$. Las formas del catalán general que presenta - $Q i$ incluso en el catalán occidental (como alegroi, etc.) tienen en ibicenco siemipre $Q \dot{i}$, resultado fonéticamente normal de - ŭ c u $1 \mathrm{u}$, con plena vitalidad, como veremos más adelante. La abertura de la $o$ en los derivados de verbos (que presentan casi todos un matiz despectivo) puede haberse producido por influencia de la $Q$ abierta del sufijo -ot.

2r. -OR (ó) < -ore formó en catalán como en latín nombres abstractos derivados de adjetivos o verbos. Actualmente parece paralizado, o por lo menos, con muy escasa fucrza creadora: abundor 'abunclancia'; bovor 'vaho'; cuor (der. de coure) 'escozor'; mollor 'gran humedad en la tierra'; picor 'escozor'.

22. -ORUM, desinencia de genitivo plural latino que, tomada de frases litúrgicas, ha servido al pueblo balear para formar derivados de tono humorístico, algo peyorativos (llatinòrum, robatónım, etc.; cfr. Moll, Gr., $\S 424)$. Parece ser típica del ibicenco la forma papussòrum 'comida'.

23. -URA <-ura. Aparte fósiles del latín (mesura, ventura, etc., del catalán general), ha formado derivados de

1 Op. cit., p. $x 60$.

2 El cambio de $Q$ en $Q$ se da en catalán en numerosos casos (nom, còdol, hora, etc.), estudiados por Joan Corominas (Algunes lleis fonetiques catalanes no observades fins ala. Estudis Romdnic.s, III, pp.6-I2), pero se trata siempre de ó en sllaba inicial. 
verbos expresanclo el resultado de la acción: garbelladures 'aechaduras'; espelladura 'rasguño, raedura', cscany'adura 'afonía'. No parece laber tenido eficacia en ibicenco para la formación de abstractos derivados de adjctivos; los abstractos que en otras regiones del catalán presentan -ura se han formado aquí con -ària (p. ej. allària) o con -or (p. ej, blavor).

24. -ÚS, -USSA $<-\overline{\mathrm{u}} \mathrm{c}$ e $\mathrm{u}$. Se da en pocos casos, sicmpre con valor peyorativo: pallis 'restos de paja'; canussa 'perro viejo y feo'.

En tartamis 'tartamudo' (catalán general tartamut) hay cambio de terminación debido probablemente a una formación regresiva sobre tartamussejar, que tiene aplicado al radical onomatopéyico el sufijo verbal -ussejar, frecuente ell catalán (ej. menjussejar). Salpis '1lovizna' (pronunciado también salpusc) parece deformación de salpuig o salpruig (<s a 1 is $\mathrm{p} 1 \mathrm{uv}$ i u m) 'salpicadura de las olas al romper cn las rocas' (Menorca).

\section{C) Surijos vivos. ${ }^{1}$}

I. Aumentativos.

a) -ÁS, -ASSA < - a c e u, - a c e a, con perfecta vitalidad: homenàs 'hombre grande'; abràs 'árbol grande'; capàs 'cabezota'; clotellìs 'pescuezo grande'; donassa 'mujer gr.'; manassa 'manaza'; bocassa 'bocaza'; dentassa 'dentaza'; gruixadàs 'muy grueso'.

1 En este capitulo se han agrupado los sufijos según su significado. Los que tienen varios valores son consignados en carla uno de los subgrupos correspondientes, pero la exposición se da conjunta $\mathrm{cn} \mathrm{cl}$ apartado de Sufijos de varios valores. Como ya se inclicó, los sufijos que tienen larga tradición en la lengua prescntan casos de especializaciones de sentido y fósiles del latin. Los que están tomados de lenguas extranjeras - muy numerosos, como se verá, y muchos de ellos no usados en el resto del dominio catalán- son seguranente de introducción moderna y no presentan concreciones en significalos especiales. 
Ventasso 'viento fuerte' (véntásu) se ha formacio a imitación del sufijo castellano - $a z o$.

Formas originariamente aumentativas que se han concretado en un sentido especial: olibassa 'lechuza', que ha desplazado al primitivo óliba en casi toda la isla; carassa 'mueca'; vidassa 'juerga, fiesta ruidosa'; dic̀s o diassa 'día' (en sentido indeterminado: $u n$ diàs, s'altra diassa 'un día').

Finalmente, queda algún fósil usado en todo el dominio lingüístico, como sedàs 'cribo' $<(\mathrm{cr}$ ib r u m $)$ s a e t a cium, pebràs > pi peraceu, 'seta'.

b) -ATXO (âsu), -ATXA. Es el sufijo italiano-accio $<-a$ c e 11 , que ha sido adoptado decididamente por el ibicenco (no se usa en el resto del catalán), en que tiene actualmente plenísima vitalidad: homenatxo, al lotatxo, abratxo, capatxo, clotellatxo, donatxa, manatxa, bocatxa, dentatxa, etc.

Siendo un sufijo tomado de una lengua extranjera, seguramente en época tardía, no presenta formas concretadas en valores especiales. La forma fardatxo 'vago, haragán' no puede considerarse como un caso de aplicación de este sufijo, sino que se trata probablemente de una extensión de significado de fardatxo 'lagarto' (dada la afición de los lagartos a tenderse al sol), forma que se cncuentra ell Valencia y parte del catalán occidental 1, y también en aragonés (Alvar, Dial. Ar. § I40); el lagarto no existe en Ibiza -actualmente, al menos-, como tampoco en Mallorca y Menorca (y es curioso que la voz propia del catalán oriental para 'lagarto', llargandaix, ha tomado en Menorca otro sentido figurado, 'grandullón, zanquilargo', ayudado sin duda por su forma fónica).

c) -ARRO, -ARRA <-*a r r u, de origen desconocido, considerado como vasco ${ }^{2}$, es vivísimo en ibicenco como en $\in 1$

1 Véase localización y estudio etimológico en JOAN COROMINEs, Mots catalans d'origen ardbic, Bullett de Dialectologia catalana, XXIV, a. 1936, pp. 19-22.

2 Cfr. Meyer-Lübke, Das Katalanische, Heidelberg, 1925, página 96, y G. RonLrs, Beitrage zur Kenntnis der Pyrenäenmundarten, RLiR, VII, I33-3. 
resto de las Baleares (mucho menos en el catalán continental) para formar aumentativos con cierto valor peyorativo: homenarro, allotarro, abrarro, caparro, clotellarro, donarra, manarra, bocarra, grossarro. No a parece concretado en significados especiales. Muy frecuente como primer elemento de la composición de dos sufijos, conservando su valor (-arran, -arrot) o habiéndolo perdido (-arró, -arri, etc.).

d) -EL.I.O, -EI. IA (éttu, ẹllé), con plena vitalidad: ho-

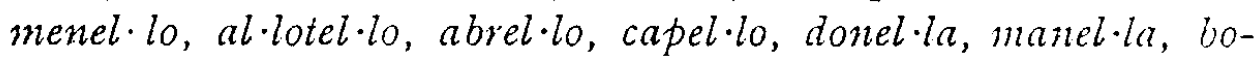
quel.la; grossel.lo ('grandote'). Su e cerrada y su valor aumentativo son exclusivos del ibicenco (en el resto de las Baleares es vivo el sufijo $-e l \cdot l o$, pero con $e$ abierta y valor diminutivo). Parece tomado del italiano -ello $<-e 11 \mathrm{u}$, de valor diminutivo. El cambio de valor tal vez sea debido a mala interpritación de un sufijo extranjero.

e) -OT, -OTA (Q́t, Q́té) <-ottu, variante de -it tu, que de un primitivo valor diminutivo pasó a diminutivo-peyorativo (conservado en francés y en catalán norte-oricntal), y en el resto del catalán, como en español, derivó hasta an1mentativo-peyorativo: homenot, al.lotot, abrot, capot, donota, manota, bocota, grossot.

Concretado en valores especiales: dacsot 'corazón de la mazorca' (de dacsa 'maíz'); barbot 'marmclla'; cassot 'blusa, chaqueta'; turissot 'oveja desde un año hasta que cría (el primer año se llama turissa)'. Es de notar que en todos estos casos se ha aplicado la forma masculina del sufijo a un nombre fenenino (el resultado es masculino, naturalmente), uso no desconocido en la aplicación actual del sufijo (un donot 'una mujerona'), aunque poco frecuente.

f) -ADA (véase Sufijos de varios valores).

g) -ER, -ERA (véase Sufijos de varios valores).

Aunque el sufijo peyorativo por excelencia es -ot, todos los aumentativos pueden usarse con sentido peronativo, excepto -ìs y -el.lo, que lo tienen muy raramente. 
2. Diminutivos.

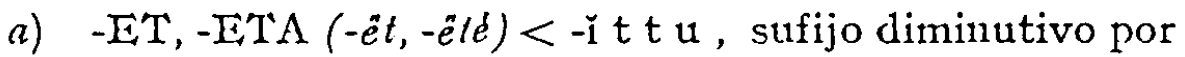
excclencia en todo el dominio catalán: homenet, al-lotet, abret, capet, clotellet, doneta, maneta, boqueta, grosset, petitet.

Han adoptado significado especial: pellet 'pelliza'; artet 'aparejo de pesca'; cossiet 'maceta'.

Aparece también aplicado a algunos radicales verbales formando nombres que indican resultado de la acción o instrumento: endevineta 'acertijo'; podet 'cuchillo curvo para podar'; brunyeta 'instrumento de zapatero, para bruñir'.

b) -Ó, -ONA (ó, ọné) <-o n e tiene en todo el dominio catalán, como en francés y provenzal, valor diminutivo, frente al aumentativo del español y el italiano ${ }^{1}$ : al lotó, clotelló; ulló (uyộ) 'ojito'; guergaló 'vientecillo del NE'; mestraló 'íd. del NO.'; bocona; manona 'manecita'. Aunque la forma femcnina -ona es viva, hay una tendencia muy acentuada a aplicar la forma -ó también a los femeninos (haciendo masculino el derivado, excepto en nombres de persona): cadiró 'sillita'; $p a$ tató; gerró 'jarrita'; marjegó 'jergón pequeño'; Esperanço 'Tsperancita’.

Aparece concretado en numerosos nombres de instrumento (en que el primitivo designa otro más grande): falçó 'tipo de hoz pequeña'; aixadó (šađộ) 'escardillo', palangró, etc.; además, dacsó 'maíz de clase pequeña'; gargamelló 'úvula'; ginebró 'fruto del enebro'; savinó 'id. de la sabina'.

Furo' 'hurón macho', que parece tener valor aumentativo, es fósil del latín $\mathrm{f} u \mathrm{r}$ on e. Parece aumentativo también fogueró 'hoguera de San Juan'; seguramente el valor afectivo ha dominado sobre el de tamaño (veremos más adelante pes-

1 Cfr. L. SpITzer, Das Suffix -one im Romanischen, en Beiträge zu romanischen Wortbildungslehre, Genève, I921, y AMADO ALONSO, Noción, emoción, acción y fantasia en los diminutivos, en Estudios lingüisticos, Madrid. Ed. Gredos, I, p. I98. 
tanyola, con sufijo diminutivo, significando 'pestañas largas', que presenta el mismo fenómeno).

La forma afarton (éfértọn) o afartasson 'glotón' cstá formada a imitación del castellano, como indica -además del valor aumentativo-peyorativo - $1 \mathrm{a}-n$ final.

c) -OI, -OIA (-ói, -óyé) <-ŭ c u $1 \mathrm{u}$. Tiene plena vida como sufijo diminutivo (cfr. -ó i en el capítulo de sufijos paralizados): homenoi, al-lotoi, abroi, capoi, clotelloi, donoia, manoia bocoia, grossoi, petitoi.

Aparece fosilizado en poi 'piojo' (grafía general poll) y genoll, usados en todo el dominio catalán (el catalán continental conserva otros, como anoll, panolla, etc., que no se usan en ibicenco).

Hay adaptación arbitraria del sufijo en rogoi, variante de rogall ('̄ugái) 'ronquera'. Xondroi 'sacudida del carro' es un caso de formación regresiva sobre xondroiar. Trempoi 'grasa con que se condimenta un cocido' deriva de tremp, deverbal de trempar.

d) -IU (iun) goza de absoluta vitalidad: homeniu; al lotin,; abriu; clotelliu; doniua; maniua; dentiua; grossin 'grandecito'; estretiu 'estrechito'.

Por su forma parece proceder del sufijo latino -i v 11 , aunque no se ve claro el cambio semántico. - I v u ha dejado en catalán numerosos fósiles usados en todo el clominio (estine, captiu, geniva, etc.) y muchos derivados románicos (festiu, ombriu, pensiu, soliu, etc. Cfr. Moll, Gr., § 4I8) raros en Ibiza, conservando todos la significación de pertenencia o analogía (salvo los derivados de verbos, que indican 'quien realiza intensamente la acción'). El cambio senántico sería, pues, el mismo producido en -i i $u$, pero éste lo sufrió ya en latín vulgar $^{1}$, y ha formado diminutivos (con más o menos j)rofusión) en todas las lenguas románicas, caso que no se da en -i v u. Quizás el cambio fué influido por el hecho de que mu-

1 Cfr. Nyrop, Gr., III, párr. 262. Cir. también Amado Alonso, loc. cit., p. 224, nota. 
chos derivados adjetivales (cfr. los citados) se prestaban a matizaciones afectivas.

Fósiles interesantes del sufijo $-\mathrm{i} \mathrm{v} \mathrm{u}$ son bassiu $<\mathrm{v}$ a c ivu 'conjunto de corderitos recién destetados' (catalán continental 'ganado destinado a ser cebado y no criar'; aragonés vacivo 'oveja que no cría' (Alvar, Dial. Ar. § I69), y bestriu 'ganado, animales (terrestres o acuáticos)', que parece proceder de *b estivu, tal vez por influencia de cabriu 'ganado cabrío' < c a privu .

e) $\mathrm{IC}$ o- $\mathrm{ICO}(i k, i k u),-\mathrm{ICA}$, de origen confuso, propio del español, especialmente del aragonés y el andaluz oriental (Alvar, Dial. Ar. § I59), ha sido adoptado por el ibicenco - presentando en su forma masculina -ic o ico indistintamente-y goza de plena vitalidad: homenic o homenico; al lotic o allotico; abrico 'arbolillo'; clotellic (o); donica; dentica ${ }^{1}$.

Aparece como primer elemento de numcrosas formas compuestas de doble diminutivo (-iquet, -iquiu, -icó), y con matiz distinto en -icot.

f) -ILLO (ílu), -ILI,A, tomado del castellano -illo < -e 11 u ha desplazado la forma autóctona -ell, hoy paralizada, y tiene en ibicenco absoluta vitalidad: homenillo, allotillo, abrillo, nasillo 'naricilla', donilla, boquilla.

Carece de formas especializadas. Cordoncillo (kuraénstlun) 'cadena de oro' e infantillo 'monaguillo' son castellanismos.

g) $-\dot{I},-$ INA $(<-i n u)$, muy vivo aún, aunque sin duda algo menos que los citados hasta ahora: al-loti, clotelli. La forma femenina -ina no es inusitada (boquina 'boquita'), pero es muchísimo más frecuente la aplicación de $-i$ al femenino: orelli, patati, terrol li.

Diminutivos concretados en significados especiales: $g a$ rroví 'grano de la algarroba'; espasi 'instrumento musical rústico, en forma de espada); jupetí (con doble diminutivo) 'cha-

1 Se usa también en el catalán pirenaico (Cfr. MOr,L, Gr., párrafo 395), con forma única -ic en el masculino. En Valencia, -ico forma diminutivos de nombres de persona: Pepico, Tonico, Vicentico, pero es muy raro en nomibres de cosa. 
leco'. Los adverbios de lugar aqui y alli han sido desplazados por las formas afectivas aquina y allina (y las dobles aquinetes, allinetes).

-i i u ha dejado numerosos fósiles usados en todo el catalán (matí, padrí, etc.), y más tarde se especializó como formador de adjetivos de materia o pertenencia (V. MIoll, Gr., $\S 407)$ y gentilicios. Este último valor es vivo aún en ibicenen, junto con el diminutivo: josepi 'hab. del pueblo de San José'; joani (zuéní) 'íd. de San Juan'; carli 'de San Carlos'.

h) -OL (-ól), -OLA (<-ŏ $1 \mathrm{u}$; cast. -uelo). Iis mucho menos vivaz que los estudiados hasta ahora, pero no puede considerarse aúl como paralizado: caminol; embatol 'brisa suave'; oratjol 'vientecillo'; llevantol; 'íd. de levante'; llebet.rol 'íd. del SO.'; covatjola 'cueva pequeña'; pestanyoles 'pestañas largas y bonitas' (el matiz afectivo desplaza aquí al diminutivo).

Sentidos especiales: barrinol 'abejorro' (derivado de barrina 'barrena'); cellola (séyélé) 'halo de la luna; espracio alargado iluminado por el sol' (de cella 'coja'); granerola 'planta de la que se hacen escobas rústicas' (de granera 'escoba'). Abellerol (béyérót) nombre de pájaro, y por extensión 'entendido en el cuidado de las abejas', es al parecer el único caso que se da en ibicenco de -ol indicando aplicación profesional o afición (V. Moll Gr. § 42I).

Quedan además numerosos fósiles propios de todo el dominio catalán: llençol, rossinyol, escarola, etc.

\section{Colectivos.}

a) - AMI ( < -a m e $\mathrm{n}$ ), vivo en todo el catalán: al lotam, cadiram, taulam, porcan 'nultitud de carne de cerdo' o 'id. de cerdoj'. Aplicado a nombres de plantas, indica multiturl de ellas $y$, naturalmente, el lugar en que se encuentran: $f i$ gueram, estepam, romaninam. De sentido más o menos concretado: relam 'raíces de un árbol'; pelam 'pelaje de un animal'. 
Numerosos fósiles del latín, de uso general: aram, eixam, llenyam, etc.

Ditam (pronunciado diktám por pseudocultismo) 'dicho, refrán', es derivado de dita 'dicho'. Aferam (éférám) (deformación de aviram) 'aves de corral', se ha concretado en 'gallinácea': una aferam 'un pollo o una gallina'.

b) $-\mathrm{AR}$ (i) (<-are), muy vivo en todo el dominio catalán, indica multitud de elenentos, o el lugar en que se encucntran: abellar (béyá) 'colmenar'; codolar 'pedregal'; familiar 'gentío'; muy usado con nombres de plantas: savinar, estepar, matar, romaninar, botjar, etc.

-a te ha formado también algún raro adjetivo de pertenencia: budell cular 'intestino recto' (en cambio en la lengua culta se mantiene vivo como sufijo adjetival: espectacular, etcétera, etc.).

Son escasos los fósiles, como collar, de uso general.

c). -UMI (<-u m en ) goza de gran vitalidad: al lotum, cadirum, taulum, cabrum; tiene con frecuencia matiz peyorativo. Es frecuente ell combinación con -er: teulerum, etc.

Muy escasos fósiles: llegım $<1$ e g u in e $n$, tenrum $<\mathrm{t}$ ener u men.

d) -ALLA (ajé) <-a 1 i a, algo menos vivaz que los precedentes, tiene con frecuencia matiz peyorativo: al-lotalla (étzutáyes); pobralla; frecuente en combinación con -er: boixeralla; al-loteralla.

Aplicado a radicales verbales expresando acción o efecto (cfr. Moll Gr., § 366) no es muy usado en Ibiza: rialla (riáye), acaballes.

e) -ER, -ERA (v. Sufijos de varios valores).

f) -ERIA (v. sufijos de varios valores).

g) -ADA (v. sufijos de varios valores).

\section{$4^{\circ} \quad$ Locativos.}

Los sufijos locativos vivos son: -AM y -AR, vistos con los colectivos, y -DOR y -ER (v. Sufijos de varios valores). 


\section{$5 .^{\circ}$ Sufijos adjetivales.}

a) $-\mathrm{A}^{\prime} \mathrm{T},-\mathrm{ADA}<-\mathrm{a} \mathrm{t} \mathrm{u}$. De su primitiva forma de participio, pasó a tener valor de sufijo adjetival, y ticne aún hoy plena vitalidad para formar (aplicado a nombres) adjctivos con significación de 'provisto de': barbat 'barbudo'; estrivancat 'mal calzado' (estrivanco significa en ibicenco 'alpargata vieja'; (blat) llevantat '(trigo) maltratado por el viento de Levante'; alicranat 'que padece de ictericia (alicrà)'.

Algunos de estos adjetivos se han sustantivado adoptando un significado especial: encanyat 'instrumento musical rústico hecho con cañas, que se hacen sonar con una castañucla'; ensegonat o enfarinat 'comida del cerdo'.

Teulat 'gorrión' es probablemente una formación regresiva sobre teuladi 'íd.' (corriente en valenciano), considerado como diminutivo.

b) -UT, -UDA ( <-utu), como -at, ha adquirido absoluta vitalidad para formar adjetivos de posesión derivados de nombres; banyut; barbut; ramut 'ramoso'; asmut 'asmático'.

Han adquirido sentido especial: garfallut 'tosco, grosero' (derivado de garfa 'garra' con doble sufijo); (fadri) garrut 'solterón' (de garra 'parte de la pierna desde la rodilla al tobillo').

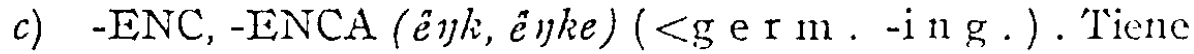
vitalidad total para formar adjetivos indicando participación o aproximación a la cualidad del primitivo: verdenc 'verdoso' (y en combinación con otros sufijos, forma aproximativos de todos los colores: blanquinenc; blavinenc; groguinenc; negrosenc; verdosenc; vermellosenc); (cel) cobertenc 'algo nublado'; homenenca 'hombruna'; (ovella) cotonenca de 'lana corta, algodonosa'; (pedra) mortenca 'que hace la cal de mala calidad' (por oposición a pedra viva). Sentidos algo especializados: penyalenc 'aficionado a escalar peñascos'; mestallcnc (méstéyë $\eta k)$ 'que tiene el cabello gris, canoso,' (de mestall 'mezcla'). Vitenc 'fuerte, robusto' deriva tal vez de vit miembro viril de cicrtos animales.' 
Aplicación especial del sufijo es la formación de gentilicios, para la que tiene plena vitalidad: eularienc 'de Sta. Eulalia'; vilenc 'de Vila (Ibiza capital)'; lletrudienc 'de Sta. Lletrudis'; santantonienc 'de Sant Antoni'.

d) -OSS, -OSA (ós, ọ́zé) (<-os u) goza de vitalidad para formar adjetivos con los siguientes usos y valores:

I. Aplicado a radicales nominales, significa 'provisto de (la cualidad del primitivo)': ginyós 'ingenioso'; empatxós 'vergonzoso'; reblós '(terreno) abundante en piedras'; ansiós 'curioso'; vetós 'que ticne rachas de humor' (de veta); diós 'vicju (:- que ticue muchos dins)'.

2. Aplicado a radicales adjetivales, significa tendencia o aproximación a la cualidad: blavinós 'azulado'; blanquinós 'blanquecino'. Salós 'que se ensucia fácilmente' se encuentra al parecer sólo en Ibiza, y parece relacionado con el francés sale, pero no se encuentra en ibicenco el primitivo correspondiente.

3. Aplicado a radicales verbales, indica el que sufre la acción del verbo - necessitós 'necesitado'- o el que la realiza o provoca: mal pensós 'mal pensado'; llenegós 'resbaladizo'; (a) Jerrissós 'pegajoso'.

c) -ER (v. sufijos de varios valores).

\section{Abstractos.}

Como indica para el francés Nyrop (Gram., III, § 111), el uso de los abstractos está fijado definitivamente en la lengua moderna. Por esto los sufijos formadores de abstractos pertenecen al grupo de los paralizados (-ança, -ença, -dat, -esa, -or, -ura). Las únicas formas vivas aún parecen ser -ERA y -ERIA (v. sufijos de varios valores). (En la lengua culta, además, los sufijos cultos: -ància, -ència, -isme).

$7^{\circ}$ Agente.

Los sufijos vivos formadores de nombres de agente son -ER, -ERA, y -DOR, -DORA, que se verán entre los sufijos de varios valores. 


\section{Instrumentos o utensilios.}

Muclios nombres de instrumentos se han formado con sufijos actualmente paralizados (-al, -all); actuahnente ticnen capacidad creadora -ER y -DOR.

\section{Acción y efecto.}

a) -MENT (mén) (<-me n t u) : aferrament, cstirament, cansament, etc. En esquadraments (éskudriméns) 'arcidas', 'náuseas' no se ve claro el radical; esquadrar significicaba en catalán antiguo 'descuartizar', lo que representa una notable diferencia semántica; en catalán oriental se usa espodrements 'arcadas', que tampoco es claro.

-Ment y -menta (del plural -me $11 \mathrm{t}$ a ) se han aplicado a algunos radicales nominales, formando derivados aunnentativos o colectivos: casament 'casa de campo'; ferramenta $<\mathrm{fe}$ r r a m e $n \mathrm{ta}$, que ha perdido el valor colectivo en su significado primario 'herramienta' (el conjunto de herramientas se llama ferramental) y lo conserva en el secundario de 'dentadura'.

b) -ADA (v. sufijos de varios valores).

Io. Sufijos de varios valores.

a) $-\mathrm{ADA}(<-\mathfrak{t a})$ tiene los siguientes usos y valores, todos ellos productivos:

I. Aplicado a radicales verbales, indica la acción expresada por el verbo, o su efecto: anada; tornada; garbejada (gérbézáảe) 'acto de transportar las gavillas'; habitada 'estancia'; fonyada 'pisotón'; furingada 'chapuzón'; tronada; nevada.

$2 .^{\circ}$ Aplicado a radicales nominales, expresa:

a) Acción intensa o golpe dado por el objeto indicado por el primitivo-boldada; codolada; quintalada; macada; llivanyada (significando todos ellos 'pedrada', según el tamaño de la piedra); mitjornada; xelocada; trammantanada 'viento fucr- 
te' (del S., del SE. y del N., respectivamente); colzada 'codazo'-, o recibido por él: clotellada, batcollada 'pescozón'.

ß) Tiene valor aumentativo-sacada 'calda fuerte' (sacu = 'caída'); alicranada 'ictericia intensa'; mal de caixalada 'fuerte dolor de muelas'-, y colectivo: cadirada 'multitud de sillas'; al.lotada 'id. de chicos'; einada 'conjunto de utensilios de cocina'; moblada 'conjunto de muebles de una casa'; hortada 'multitud de huertos'.

$\gamma$ ) Indica la acción propia del individuo indicado por el primitivo: al.lotada; asenada 'burrada'; carallada (kéréyádé) 'pillada'.

Con especialización de significado: jomalada 'trabajo intenso'; setmanada 'paga de una semana'; ventrada 'conjunto de tripas del cerdo' o 'conjunto de animales nacidos de un solo parto'; llanada 'pincel de lana para alquitranar la barca'. Ha perdido el valor colectivo en plomada y surada 'cada uno de los plomos o de los corchos de una red', y el valor intensivo en bandada 'lado'.

Presenta fósiles del latín, como aixada, ferrada, vegada, etcétera, de uso general.

a) -DOR, -DORA (dọ, dọ́ré) (resultante de los sufijos latinos -tore $y$-t o $r i u$ ) tiene plena vitalidad para formar:

a) nombres $y$ adjetivos de agente o de instrumento: flastomador 'blasfemador'; afogador (éfugéd̄ọ) 'derrochador'; pegellador 'inspector de pesos'; esgarinzador 'columpio'; furgador "hocico del cerdo'; aplegador 'pala para recoger barreduras'.

ß) Nombres que indican el lugar en que se realiza la acción: dormidor 'barra en que duermen las gallinas'; ponedor 'lugar en que ponen los huevos'; batedor (de sol) 'lugar en que da el sol con toda intensidad'. [La forma culta derivada de -toriu, -tori, se encuentra, aparte los cultismos (reclinatori, etcétera), en una curiosa forma popular: corretori corrida, carrera'].

c) -ER, -ERA (é, éré), <-a I i u, -a ri a, sufijo latino de gran vitalidad para la formación de adjetivos, muchos de ellos sustantivados ya en latín (que han dado fósiles de uso 
muy general, como caldera, carrer, gener, etc., etc.). Esto hizo que se mantuviera en las lenguas románicas como sufijo adjetival y como formador de sustantivos, en dos formas, masculina y femenina, y con una serie de valores, todos los cuales tienen aún hoy plena vitalidad. Como en la mayoría de los casos los derivados de la forma masculina y los de la femenina tienen idéntico valor, conviene estudiarlos aquí juntos, indicando los usos que son exclusivos de la forma fcncuina.

I. ${ }^{\circ}$ Forma adjetivos indicando cualidad, pertencucia 0 parecido: (pedra) foguera 'pedernal'; (peix) roquer 'pez que se encuentra junto a las rocas'; (peix) fanguer 'id. en el fango'; feiner 'laborioso' (en cambio, dia fener 'día laborable', derivado de la forma arcaica faena, usada aún en Valencia); (terra) pebrassera 'que produce muchas setas'; fogater 'fácilmente combustible'. Concretados en sentido especial: ratoner 'avaro, regatero' (deriv. de rata); (conill) maner 'concjo doméstico' (deriv. de má) ; falaguer (< árabe halaka 'poner liso') 'de mala calidad; indispuesto, enfermo' (en cambio, en el resto del dominio catalán significa 'agradable, bien'. "No me encuentro biea» es en Ibiza Estic falaguer, y cn Mallorca: No estic falaguer).

2. Como formativo de nombres, tiene los siguientes usos y valores:

A) Aplicado a radicales nominales, indica:

a) La persona dedicada profesionalmente o con afición al objeto indicado por el primitivo: correner 'cartero rural'; escarader 'destajista'; animer 'encargado de pasar el cepillo de las ánimas en la iglesia'; maquinera 'mujer que hace medias a máquina'.

B) El lugar en que se encuentra el objeto: sitger 'solar de la carbonera'; torrentera 'lecho del torrente'; paller (péyé) 'pajar (al aire libre)'; pallera 'pajar (cubierto)'.

r) Utensilio o instrumento: greixonera 'cazucla' (deriv. de greix 'grasa'); enciamera 'plato grande para ensalada'; $p a$ langrer 'barca que pesca con palangres'; gambaner 'gambarón'.

ס) Planta productora del fruto designado por el primitivo: llimoner; bellotera 'encina'; dacsera 'planta del maíz'; cs- 
cartera 'clase de cardo'; trapanera (trépénẹré) 'alcaparrera' (de taparera, con deformación del radical). Pebrera ha pasado a significar 'pimiento' (catalán pebre), y la planta se llama pebrevera.

ع) Aumentativo o colectivo: verder 'abundancia de (hierba) verde'; rosadera 'rocío abundante'; cadarnera 'resfriado fuerte' (deriv. de cadarn); platera 'plato grande, fuente'; ramera 'ramaje'; casera 'nido de abejas'; guesper 'avispero' (de gues$p a$ 'avispa', al parccer exclusivo del ibicenco -cfr. francés guêpe-; en el resto del catalán es vespa o vespra); caixalera 'dentadura'; genivera 'encía'.

ל) Gentilicios: vilaner 'de Vila'; lletrudier 'de Sta. Lletrudis'; caler 'de Sa Cala'; carler o carliner 'de Sant Carlos'; coroner 'de Corona'.

n) Objeto que tiene la cualidad del primitivo o es propio de él: creuer 'radio de la rueda'; vorera 'dobladillo de un vestido' (de vora 'borde'); galteres 'paperas'.

B) Aplicado, en su forma femenina, a radicales adjetivales, forma abstractos de cualidad: sordera, gatera, borratxera.

C) Aplicado, también en su forma femenina, a radicales verbales, forma abstractos indicando necesidad o deseo intenso de realizar la acción: gitera 'ganas de vomitar' (de gitar < i a ctare), badallera 'íd. de bostezar'; correra 'id. de correr'. Para unirse a los verbos en -ir y -re se refuerza con -ec: cosiguera 'ganas de coser', escupiguera. Ha formado también algún nombre de instrumento: suera 'manta del caballo' 1.

1 La forma culta de -a ri u es en catalán -ari, que se encuentra en ibicenco en cultismos y en el adjetivo revessari (deriv. de revés) 'extraño, fuera de lo corriente; impropio' (paraules revessdries 'palabras extrañas', el comer carne en Cuaresma es revessari, etc.).

La forma -ària para abstractos de dimensión, propia del catalán y del sardo (que MFYLR-I,ÜBkE, Gr., II, pírr. 470, considera de origen inexplicado, y MoI.L, Gr., párr. 372, como probable cultisıno) se usa en ibicenco en los misuros casos que en el resto del catalín: llargdria, amplaria, fondiria, altària (y también altiria, mezcla de altdria y altura). 
d) -ERIA (érié), como en francés y en español, lia desplazado a -ia para la formación de derivados. Es nuly productivo, aunque bastante menos que su correspondiente francés-erie. Sus usos y valores son:

I. Aplicado a radicales nominales o a adjetivos substantivados, forma nombres con valor de abstractos de cualidad y de acción propia del individuo indicado por el primitivo: beneiteria, aseneria, dolenteria, al loteria.

2. Aplicado a nombres, tiene valor colectivo: caixoneria 'conjunto de cajones'; cadireria 'íd. de sillas'; al-loteria 'íd. de chicos'. Partiendo de este sentido colectivo, sirve para designar el local en que se vende o se trabaja el objeto indicado por el primitivo: fusteria 'carpintería'; espardenyeria 'alparsatería'; forneria 'panadería' se ha formado a imitación de éstos (forn, usado en el catalán general para 'panadería', designa sólo el horno familiar, que se encuentra en casi todas las casas; sólo existen algunas panaderías en Ibiza capital).

\section{ACUmulación DE SUFijos}

La formación de derivados con dos o más sufijos (ej. esp. carretón, callejón, chiquirritin, grandullón) es siempre muy usada en el habla popular (lo mismo que la abundancia de sufijos de valor idéntico); con todo, tal vez sea el ibicenco el habla catalana que más uso hace de estas formaciones. Por lo menos, no hay duda que los ibicencos usan y abusan mucho más de las formas derivadas que los otros habitantes de las Baleares.

El significado de una forma derivada con dos o más sufijos, corresponde siempre al valor del último de los componentes; en cambio, el valor del primer componente varía seguin los casos, por lo que se pueden clasificar estas formas polisufijadas en los siguientes grupos:

I. El primer sufijo es inoperante (y por io tanto, en estos casos, muerto, aunque tenga vitalidad como sufijo indepondiente), hasta el punto de que un sufijo aumentativo como 
-arro aparece en composición con otro diminutivo $(-i,-\delta)$, teniendo el resultado valor puramente diminutivo; el conjunto de los dos sufijos aparece, pues, como uno solo, que tiene valor idéntico al del último elemento. Estos compuestos son numerosos: -arri, -arret, -arró, -arrell: boscarri 'bosquecillo', nassarri 'naricilla', venarrina 'vocecilla', panxarrina 'barriguita'; fangarret 'barro (en poca cantidad)'; nassarró 'naricilla', caparro 'cabecita'; mocarrell 'mocos'; -arrera: llengarrera 'ganas de hablar'; -arrut: caparrut 'testarudo', forçarrut 'forzudo'.

-egut: forcegut 'forzudo'; -egada: fartegada 'hartazgo', maregada 'marejada', ventegada 'vendaval'; -egar: ventegar 'vendaval', pedregar 'pedregal'.

-etut: aletut 'aludo', -etada: llametada 'relampagueo intenso', peixetada 'multitud de peces o de pescado'; -eter: femeter 'basurero'.

-erut: geperut 'giboso', llarguerut 'larguirucho'; -erell: fumerella 'columnita de humo'.

-ellut: camellut 'zancudo', gropellut 'tosco, rudo'.

-olenc: fredolenc 'frío, casi frío'.

-assós: boirassós (buirésọs) 'ligeramente nublado'.

2. El primer sufijo tiene el mismo valor que el segundo, por lo que vienen a reforzarse mutuamente: a) Dobles diminutivos: al-lotiquet, al-lotiquiu, al-lotico; homeniquet, homeniquiu, homenico; al-lotillet, al.lotillo; maniueta 'manecita', pineto 'pinillo', carreto ; ventolina 'vientecillo', tanconet 'cercadi11o'. b) Dobles aumentativos: pinyarrot, penyalot, donarrassa, pitarrds, camarrassa, caparrot. c) Dobles colectivos: al.loteralla, boixeralla, frarumer (deriv. de frare 'clase de oruga'), xerrumer 'parloteo', rajumer 'chorro' (deriv. de raig), teuleram, teulerada, teulerum (los tres, 'conjunto de tejas'). Vellerum (véyérum), en principio 'conjunto de viejos', se ha individualizado: un vellerum 'un viejo, un carcamal'.

3. El primer sufijo tiene valor diferente del segundo $\mathrm{y}$ manticne ese valor, a pareciendo el resultado como derivado de otro derivado: belloterar 'encinar', herbatjar 'abundancia de herbaje', ferramental 'conjunto de herramientas', formiguerada 'íd. de hormigueros', forfolladissa 'manoseo', remenadissa 
'meneo, movimiento reiterado'; caseró 'casilla del nido de abcjas'; cimerol 'rama que sobresale del árbol'.

4. El matiz afectivo del primer clemento domina sobre cl segundo, y el derivado presenta mezcla de ambos matices; es lo que ocurre principalmente en las formas compuestas -icot y-otell: al loticot 'muchacho ya crecido', jovenicota 'muchacha', belicot 'lloro' (todos ellos con claro matiz de simpatía); garbotell 'gavilla pequeña y de mala calidad', espigotell 'espiga mala o mal trillada', al lotell 'chicuelo'.

Formaciones con triple sufijo: Por lo general, el primer elemento no juega papel alguno, y el segundo sufijo tiene valor idéntico al tercero, o diferente (y en cste caso lo conserva): mocarrellera, mocarrellada 'mocarro', pelagassera 'gran abundancia de pelo', polseguerada 'íd. de polvo', fumarcllacia 'íl. de liumo', tenlerería 'íd. de tejas', etc., etc.

Todo lo expuesto da idea de la riqueza de recursos del ibicenco. Su carácter insular y las actividades marineras de muchos de sus habitantes han determinado la fisonomía especial del dialecto y la adopción de sufijos y de voces extranjeras no usadas, o raras, en el resto del dominio catalán. Por lo demás, tanto en los rasgos fonéticos y morfológicos como en el léxico, el ibicenco concuerda tan pronto con cl catalán central como con el valenciano o con el resto del baleárico, presentando en conjunto un panorama variado y tiquísino, apasionante para el lingüista pero abundante en problemas, a veces de difícil solución.

Ana Moll. 